\title{
PRIMERA CONTRIBUCION AL CONOCIMIENTO DE LOS PLECOPTEROS DE GALICIA: LA CUENCA DEL TAMBRE
}

\author{
P. Membiela \\ I.N.B. "Otero Pedraio" Orense \\ Palabras Clave: Plecoptera, Tambre river (Galicia)
}

\begin{abstract}
FIRST CONTRIBUTION T O T HE KNOWLEDGE OF T HE PLECOPTERA OF GALICIA: THE TAMBRE BASIN.

We point out the capture of 22 species of Plecoptera in the Tambre basin, and we have to emphazise the presence of Isoperla grammatica sp. III sensu Berthélemy, 1979 first record for the area 1 of the Limnofauna Europaea (Illies, 1978) and Leuctra lusitanica Aubert, 1962 only cited in Portugal and also the capture of several specimens of Leuctra maroccana Aubert, 1956 which had been cited as a transgressant species for the area 1 of the Limnofauna Europaea (op. cit.).

We bring forward new data to the knowledge of the distribution of the Plecoptera in the Iberian Peninsula, which support the scheme proposed by Berthélerny and Terra (1980).

Finally, we describe the vertical distribution and the flight periods of the different species:
\end{abstract}

\section{INTRODUCCION}

El presente trabajo representa el primero de una serie de estudios que hemos emprendido cara al conocimiento de diversos aspectos taxonómicos, biológicos y ecológicos de los Plecópteros de Galicia.

Los únicos datos de que se disponía hasta la fecha se reducen a unas pocas citas aisladas dentro de trabajos más amplios no dedicados específicamente al estudio de esta zona (ver Aubert, 1963; Zwick, 1972; Berthélemy y Terra 1980).

En este estudio se presenta un catálogo de los Plecópteros de la cuenca del Tambre y se aborda el conocimiento de la distribución altitudinal y de los períodos de vuelo de las especies inventariadas.

El principal interés de estos trabajos reside en el escaso conocimiento que existe sobre muchas de las especies capturadas, debido al elevado endemismo que presenta la parte noroccidental de la Península Ibérica, zona en la que se halla localizada nuestra área de muestreo.

\section{EL MEDIO Y METODOS DE ESTUDIO}

1.- Características generales de la cuenca del Tambre

La cuenca del Tambre comprende una superficie aproximada de $1500 \mathrm{Km}^{2}$. El río Tambre se considera que nace en la Laguna de Sobrado dos Monxes a 510 m. de altitud. El río discurre en dirección E-W hasta su desembocadura en la ría de Muros-Noia tras un recorrido de unos $139 \mathrm{Km}$. Los principales afluentes son el Samo, el Maruzo y el Lengüelle por la orilla izquierda y el Mera por la derecha (Fig. 1).

La cuenca del Tambre está formada por granitos, rocas básicas y metamórficas; como consecuencia los suelos son ácidos predominando las tierras pardas.

La vegetación es variable presentando zonas de tojales u otros tipos de monte bajo junto con otras áreas cubiertas por pinares o robledales, tampoco faltan las zonas de cultivo.

El clima está dominado por un componente oceánico, la prec pitación anual oscila entre 1400 y 
$1900 \mathrm{~mm}$ y la amplitud térmica media anual se aproxima a $11^{\circ} \mathrm{C}$ en toda la cuenca. El mes más caluroso corresponde a julio y el más frío es enero.

\section{2.- Estaciones y métodos de muestreo.}

Se han realizado capturas de adultos y exuvias de Plecópteros en un total de 9 puntos en la cuenca del Tambre. Siete de ellos sobre el río Tambre (Estaciones 1, 4, 5, 6, 7, 8 y 9), uno en el río Mera (Est. 3 ) y uno en el río Batán (Est. 2).
Corredoiras. Lat. N: 43ำ 1'; Long. W: $8^{\circ} 3^{\prime}$; Alt: 480 m. Est. 3.- Río Mera: puente en la carretera cerca de Orxal. Lat.N: 420 58'; Long. W: $8^{\circ} 3^{\prime}$; Alt: 460 m. Est. 4.- Río Tambre: Gosende. Lat. N: 48Q 3' 30"; Long. W: 80 10' 30"; Altitud: $320 \mathrm{~m}$.

Est. 5.- Río Tambre: Pontecarreira. Lat. N : $43^{\circ}$ 1' 20"; Long. W: 80 15' 20"; Altitud: $280 \mathrm{~m}$.

Est. 6.- Río Tambre: Sigüeiro (antes del pueblo). Lat. $\mathrm{N}$ : 420 58' 0"; Long. W: 8 25' 50"; Altitud: $230 \mathrm{~m}$. Est. 7.- Río Tambre: Sigüeiro (después del pueblo).

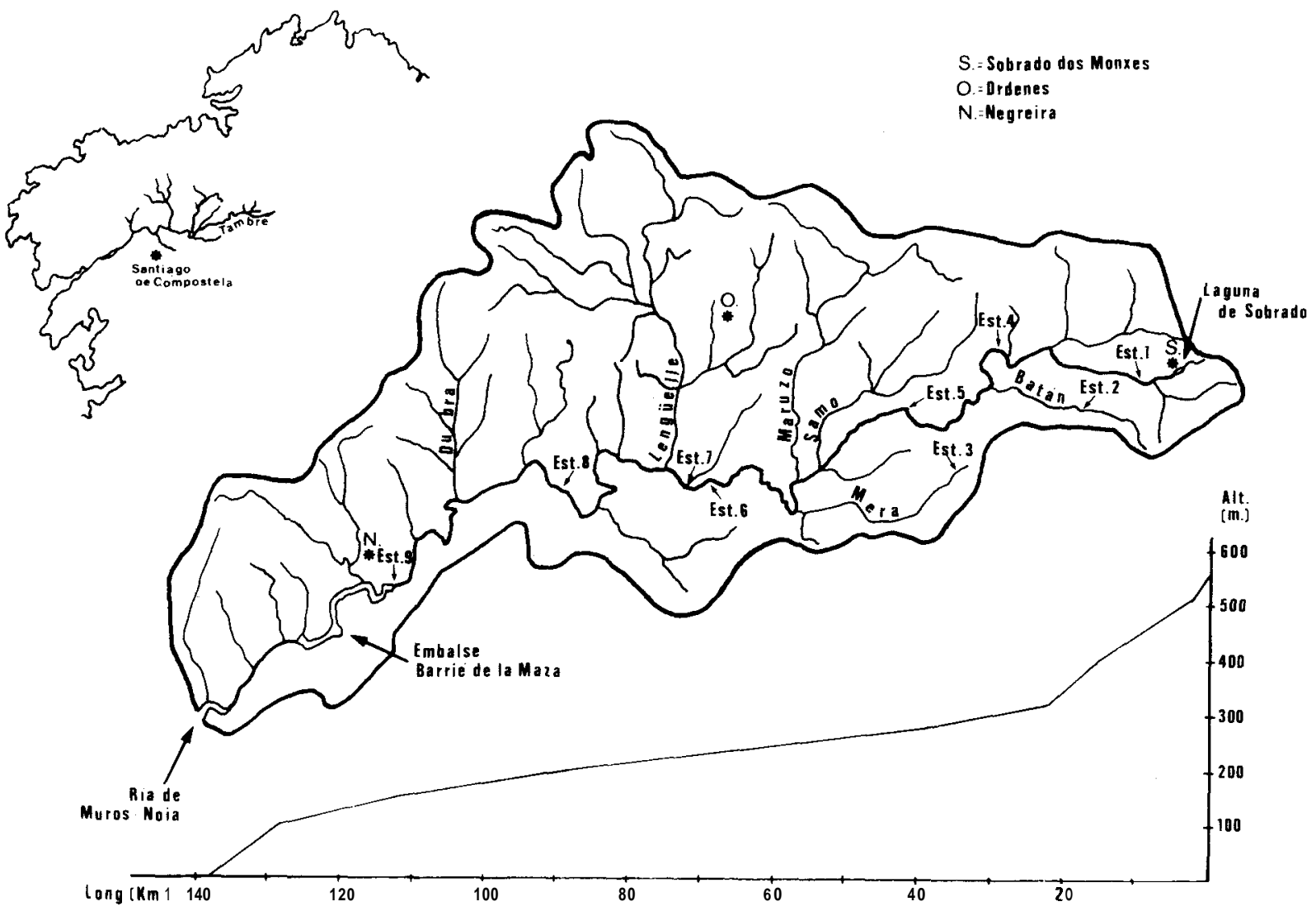

Fig 1 . Cuenca del Tambre red hidrográfica y perfil del río

Tambre Basin river system and profile of the river

Las recogidas se han efectuado con una periodicidad al menos mensual entre los meses de febrero y diciembre de 1982 y de manera más esporádica durante el año 1979.

A continuación enumeramos las estaciones muestreadas señalando para cada una de ellas sus coordenadas y altitud.

Est. 1.- Río Tambre: a $3 \mathrm{Kms}$ aguas abajo de Sobrado. Lat. N: $43^{\circ} 1^{\prime} 50^{\prime \prime}$ Long. W: 80 2' 0"; Altitud: 480 m. Est. 2.- Río Batán: puente en la carretera de Sobradoa
Lar. N: 420 57' 50"; Long. W: 8²7’ 0"; Altitud: 230 m. Est. 8.- Río Tambre: Ponte Albar. Lat. N : 42@ 57’ 50"; Long. W : 8 $33^{\prime}$ 40"; Altitud: $200 \mathrm{~m}$.

Est. 9.- Río Tambre: Ponte Maceira. Lat. N : 42o 54' 10"; Long. W : 8o 42' 10"; Altitud: $150 \mathrm{~m}$.

\section{RESULTADOS Y DISCUSION}

Relación de especies 
A lo largo del presente estudio han sido capturados un total de 658 adultos (148 machos y 310 hembras) y 116 exuvias. Este conjunto está representado por 5 familias, 10 géneros y 22 especies y subespecies.

En este apartado presentamos un inventario de las distintas especies y subespecies señalando para cada una de ellas los siguientes datos:

La fecha y el número de individuos encontrados en cada estación mediante los símbolos convencionales: machos $(\mathrm{m})$, hembras $(\mathrm{h})$ y exuvias (e).

En caso de que la especie sea nueva para Galicia, España o la Península Ibérica se citará esta circunstancia.

Familia Taeniopterygidae

1.-Taeniopteryx scboenemundi (Mertens, 1923)

Est. 6: 24-1-79, le; Est. 9: 3-2-79 1e. Total: 2e

Familia Nemouridae

2.- Protonemura globosa Berthélemy y Terra, 1980 Est. 1: $15-5-82,3 \mathrm{~m} ; 8-7-82,1 \mathrm{~m}$ y $1 \mathrm{~h} ; 7-9-82,1 \mathrm{~m}$; 19-9-82, 1m; 2-10-82, 1m; 28-11-82, 1h; Est. 2: 15-5-82, lm y $1 \mathrm{~h} ; 6-6-82,1 \mathrm{~h} ; 8-7-82,1 \mathrm{~h} ; 7-9-82$, 1h; 19-9-82, 1m; 2-10-82, lh. Est. 3: 28-3-82, 1h; 6-6-82, $1 \mathrm{~m}$ y $3 \mathrm{~h} ; 30-6-82,1 \mathrm{~m}$ y $1 \mathrm{~h} ; 19-8-82,1 \mathrm{~m}$ y $1 \mathrm{~h}$; $7-9-82,2 \mathrm{~m} ; 19-9-82,1 \mathrm{~m}$ y $1 \mathrm{~h}$. Total $15 \mathrm{~m}$ y $14 \mathrm{~h}$.

3.- Protonemura hispanica Aubert, 1956

Est. 1: 14-10-79, 2m;19-9-82, 2m;2-10-82, $7 \mathrm{~m}$ y $8 \mathrm{~h} ; 24-10-82,1 \mathrm{~m}$ y $3 \mathrm{~h}$. Est. $2: 2-10-82,5 \mathrm{~m}$ y $7 \mathrm{~h}$. Est. 3 : 19-9-82, $1 \mathrm{~m}$. Total: $18 \mathrm{~m}$ y $18 \mathrm{~h}$.

Primera cita para Galicia.

4.- Protonemura mejeri Pictet, 1842

Est. 1: 28-2-82, le. Est. 3: 28-3-82. 2h. Est. 4: 16-4-79, 1h; 18-4-82, 1h. Est. 5: 28-1-79, 1h; 28-2-82, 1m. 28-3-82, 2h. Est. 6: 24-1-79, 1h. Est. 7: 16-4-79, 1h. Total: $1 \mathrm{~m}, 9 \mathrm{~h}$ y le.

Primera cita para Galicia.

\section{5.- Protonemura pyrenaica asturica Aubert, 1954}

Est. 1: 28-2-82, 1h; 18-4-82, 4e; 6-6-82, 1h. Est. 2: 18-4-82, $5 \mathrm{~m}$ y $4 \mathrm{~h} ; 16-5-82$, 1h. Est. $3: 28-3-82,1 \mathrm{~h}$. Total: $5 \mathrm{~m}, 8 \mathrm{~h}$ y $4 \mathrm{e}$.

Primera cita para Galicia.

6.- Amphinemura sulcicollis guadarromensis Aubert, 1952
Est. 1: 16-5-82, 4h; 6-6-82, $1 \mathrm{~m}$ y Ih. Est. 2- 16-5-82, $1 \mathrm{~h} ; 6-6-82,1 \mathrm{~m}$. Total: $2 \mathrm{~m}$ y $6 \mathrm{~h}$.

Primera cita para Galicia.

7.- Nemoura ceciliae Aubert, 1956

Est. 2: 24-10-82, 2h. Total: $2 \mathrm{~h}$

Primera cita para Galicia

8.- Nimoura cinerea umbrosa E. Pictet, 1865

Est. 1: 13-5-79, 3h. Est. 4: 16-4-79, $1 \mathrm{~m}$. Total: $1 \mathrm{~m} \mathrm{y}$ $3 h$.

Familia Leuctridae

\section{9.- Leuctra franzi Aubert, 1956}

Est. 1: 30-8-79, $1 \mathrm{rn} ; 11-9-79,6 \mathrm{~m}$ y 14h; 14-10-79, $1 \mathrm{~m} \mathrm{y} 1 \mathrm{~h} ; 19-8-82,1 \mathrm{~m} ; 8-9-82] ,1 \mathrm{~m}$ y $20 \mathrm{~h} ; 19-9-82,7 \mathrm{~m}$ y $14 \mathrm{~h} ; 2-10-82,4 \mathrm{~m}$ y $14 \mathrm{~h}$. Est. $2: 8-9-82,4 \mathrm{~m}$ y $2 \mathrm{~h} ; 19-9$ $82,3 \mathrm{~m}$ y $2 \mathrm{~h} ; 2-10-82,5 \mathrm{~h} ; 24-10-82,1 \mathrm{~m}$. Est. 3: 22-782, 1 h. Est. $14-10-79,1 \mathrm{~h} ; 8-9-82,2 \mathrm{~m} ; 19-9-82,2 \mathrm{~m}$. Total: $43 \mathrm{~m}$ y $74 \mathrm{~h}$.

Primera cita para Galicia.

10.- Leuctra fus ca Linné, 1758

Est. 1: 2-10-82, 1h; 24-10-82, 1h. Est. 4: 14-10-79, $2 \mathrm{~m}$ y 4 h. Est.5: 14-10-79, 1h. Est. 7: 14-10-79, Im. Est. 9: 19-10-79, 1h. Total: $3 \mathrm{~m}$ y $8 \mathrm{~h}$

Primera cita para Galicia.

11.- Leuctra geniculata Stephens, 1835

Est. 1: 2-10-82, 1h. Est. 4: 14-10-79, $4 \mathrm{~m}$ y $5 \mathrm{~h} ; 2-10$ 82, 1 h.Est. $5: 30-10-79,1 \mathrm{~m} ; 14-10-79,2 \mathrm{~m}$ y $1 \mathrm{~h} ; 19-10$ 82, $1 \mathrm{~m} ; 2-10-82,1 \mathrm{~m}$. Est. 6: 14-10-79, $1 \mathrm{~m}$ y 1 h. Est. 9: 14-10-79, $1 \mathrm{~m}$. Total: $11 \mathrm{~m}$ y $9 \mathrm{~h}$.

Primera cita para Galicia.

\section{2.- Leuctru hispanica Aubert, 1952}

Est. 1: 14-10-79, 2m;8-9-82, 1m;19-9-82, $2 \mathrm{~m}$; $2-10-82,7 \mathrm{~m}$ y $15 \mathrm{~h}$. Est. $2: 19-9-82,4 \mathrm{~m} ; 2-10-79,10 \mathrm{~m}$ y 13h. Est. 3: 2-10-82, Ih. Est. 5: 2-10-82, $1 \mathrm{~m}$. Total: $18 \mathrm{~m} \mathrm{y} 29 \mathrm{~h}$.

Primera cita para Galicia.

13.- Leuctra lusitanica Aubert 1962

Est. 1: 24-10-82, 1h. Total: $1 \mathrm{~h}$.

Primera cita para España.

14.- Leuctru madritensis Aubert 1952

Est. 1: 14-10-79, $1 \mathrm{~m} ; 2-10-82,23 \mathrm{~m}$ y $13 \mathrm{~h} ; 24-10-82$, $13 \mathrm{~m}$ y $15 \mathrm{~h}$. Est. 2 : $2-10-82,1 \mathrm{~m}$ y $2 \mathrm{~h} ; 24-10-82,8 \mathrm{~m}$ y 
18h. Total $46 \mathrm{~m}$ y $48 \mathrm{~h}$.

Primera cita para Galicia.

\section{5.- Leuctra maroccana Aubert, 1956}

Est. 1: $28-11-82,25 \mathrm{~m}, 8 \mathrm{~h}$ y $8 \mathrm{e} ; 7-12-82,14 \mathrm{~m}$ y $13 \mathrm{e}$ Est. 2: $28-11-82,17 \mathrm{~m}$ y $2 \mathrm{~h} ; 7-12-82,46 \mathrm{~m}$ y $24 \mathrm{~h}$. Est. 3 : 28-3-82, 1h. Est. 4: 28-11-82, 6m y 4h. Est. 6: 24-1-79, $1 \mathrm{~m}$. Total: $109 \mathrm{~m}, 44 \mathrm{~h}$ y $21 \mathrm{e}$.

Primera cita para Galicia.

16.- Leuctra stupeningi Illies, 1954

Est. 4: $27-1-79,1 \mathrm{~h}$ y le. Total: $1 \mathrm{~h}$ y le.

Primera cita para Galicia

Familia Perlodidae

17.- Isoperla bipartita Aubert, 1962

Est. 4: 10-8-79, $1 \mathrm{~m}$. Total: $1 \mathrm{~m}$.

18.- Isoperla grammatica sp. II sensu Berthélemy, 1979

Est. $1: 3-6-79,1 \mathrm{~h} ; 16-5-82,2 \mathrm{~m}$ y $1 \mathrm{~h} ; 6-6-82$, $4 \mathrm{~m}$ y $1 \mathrm{~h}$ y $4 \mathrm{e} ; 22-7-82,2 \mathrm{~m}$ y $1 \mathrm{~h}$. Est. 2: 22-7-82, $1 \mathrm{~m}$. Est. 3: 6-082 , Ih. Est. 4: 16-5-82, $1 \mathrm{~m} ; 6-6-82,2 \mathrm{~m}, 4$ h y le; 30-6$82,1 \mathrm{~h} ; 8-7-82,3$ e. Est. 5: 6-6-82, 2e. Total: $12 \mathrm{~m}, 10 \mathrm{~h}$ y $10 \mathrm{e}$.

Primera cita para la región 1 de la Limnofauna Europaea (Illies, 1978).

19.- Perlodes dispar Rambur, 1842

Est. 1: 18-4-82, 6e. Est. 4: 21-3-79, 1h; 27-2-82, 2e; 28-3-82, 7e. Est. 5: 30-6-82, 2e. Total: 1 h y 17 e.

Primera cita para España.

Familia Perlidae

20.- Martbamea rp.

Est. 5: 24-6-79, $1 \mathrm{~h} ; 2-7-79,1 \mathrm{~h}$. Total: $2 \mathrm{~h}$.

\section{1.- Perla madritensis Rambur, 1842}

Est. 1: $18-4-82$, le; $16-5-82,7 \mathrm{~m}, 1 \mathrm{~h}$ y le; 6-6-82, $3 \mathrm{~m}, 1 \mathrm{~h}$ y $19 \mathrm{e}$. Est. 2: 6-6-82, le. Est. 3: 30-6-82, $1 \mathrm{~m}$. Est. 4: $10 \mathrm{~m}$ y $2 \mathrm{~h} ; 6-6-82,15 \mathrm{~m}, 1 \mathrm{~h}$ y $21 \mathrm{e} ; 30-6-82,5 \mathrm{~m}$; 8-7-82, 1e. Est. 5: 6-6-82, 12e; 30-6-82, $1 \mathrm{~m}$. Total: $42 \mathrm{~m}, 5 \mathrm{~h}$ y $56 \mathrm{e}$

\section{2.- Siphonoperla torrentium Pictet, 1842}

Est. $1: 3-6-79,1 \mathrm{~m} ; 24-6-79,2 \mathrm{~m}$ y $1 \mathrm{~h} ; 16-5-82,3 \mathrm{~m} \mathrm{y}$ $3 \mathrm{~h} ; 6-6-82,4 \mathrm{~m}, 3 \mathrm{~h}$ y $2 \mathrm{e} ; 8-7-82,2 \mathrm{~m}$ y 2 h. Est. $2: 18-4$ 82- $1 \mathrm{~m} ; 16-5-82,1 \mathrm{~m}, 4 \mathrm{~h}$ y le; $22-7-82$, 1m. Est. 4: 11 $9-79,1 \mathrm{~h} ; 16-5-82,3 \mathrm{~m} ; 6-6-82,1 \mathrm{~h}$ y $1 \mathrm{e} ; 30-6-82,3 \mathrm{~m}$ y

\section{3h. Total $21 \mathrm{~m}, 18 \mathrm{~h}$ y $4 \mathrm{e}$}

Tan solo cinco de ellas (T.schoenemundi, P. globosa, I. bipartita y P. madritensis) habían sido ya previamente citadas en el país gallego por otros autores (ver Zwick, 1972 y Berthélemy y Terra, 1980).

\section{ESTUDIO BIOGEOGRAFICO}

Se observa mucha mayor similitud entre la cuenca del Tambre y las Cordilleras Ibérica, Montes Cantábricos y Cordillera Transversal (Guadarrama, Gredos, Gata y Estrela), que entre aquélla yel resto de la Península Ibérica, Pirineos incluídos.

Nuestros datos apoyan el esquema biogeográfico propuesto por Berthélemy y Terra (1980) que consideran solamente tres tipos de repartición para la Península Ibérica frente al esquema original de Aubert (1963) que definía un número muy superior, de hecho apenas hay diferencia entre el poblamiento de la Cuenca del Tambre yel de las regiones limítrofes (Cordillera Cantábrica y Cordillera Transversal).

De acuerdo con este criterio podemos considerar que del total de 22 especies y subespecies encontradas se pueden considerar a 11 de ellas como nordibéricas (P. globosa, P. hirpanica, P. p. asturica, A. . guadarramensis, N. ceciliae, $L$. franzi, L. hispanica, $L$. lusitanica, $L$. madritensis, $L$. stupeningi $y \quad P$. mudritensis) aunque 3 de ellas (P. globosa, A. s. guadarramensis y L. franzi) han sido incluídas de manera provisional dentro del grupo nordibérico debido a las deficiencias existentes en el conocimiento de su distribución. Un segundo grupo está constituido por las especies europeas que suman en total 8 ( $\mathrm{T}$. schoenemundi, P. meyeri, L. fusca, L. geniculatu, I. grammatica sp. III, P. dispar, Martbamea sp. y S. torrentium) y por último dos especies (L. maroccana $e I$. bipartita) serían consideradas como meridionales. La escasez de datos nos impide pronunciarnos sobre el encuadramiento de la subespecie N. c. umbroi-u.

\section{DISTRIBUCION ALTITUDINAL}

En la fig. 2 se presenta la repartición altitudinal de las diferentes especies y subespecies encontradas en la cuenca del Tambre.

Las diferencias de altitud son demasiado pequeñas para poder observar una clara sustitución de unas 
especies por otras a lo largo de la cuenca. Noobstante podernos deducir que la mayoría de los taxa se encuentran representados en la cuenca alta y media del río Tambre y solo algunos de ellos (P.meyeri. L. fuscata, L. geniculata y L. maroccana) pueden colonizar la cuenca baja. Una sóla especie (T. schoenemundi) presenta una clara preferencia por las zonas bajas de la cuenca. Un fenómeno similar ha sido señalado por Berthélerny y Laur (1976) en el río Lot.

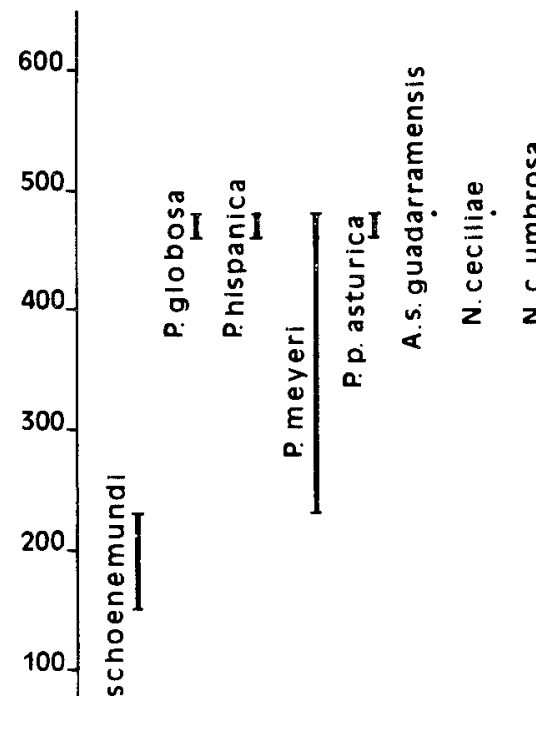

3.- Especies otoñales

Dentro de este grupose encuentran P. bispanica. L. franzi. L. fusca. L. geniculata. L. bispanica, L. madritenus.

4.- Especies con período de vuelo de larga duración Sólo la especie P. globosa presenta un período de vuelo de larga duracihn

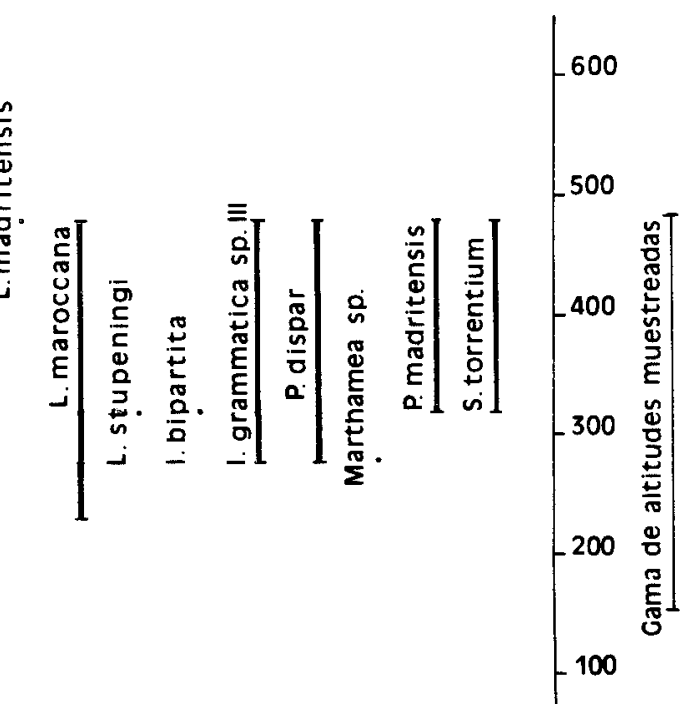

Figurd 2- Distribución altitudinal de los Plecópteros en la cuencia del Tambre

Vertical distribution ot the Plecoptera in the Tambre basin

\section{PERIODOS DE VUELO}

Presentamos los periodos de vuelo de las diferentes especies de la cuenca del Tambre en la fig. 3.

A la vista de los datos podemos deducir la existencia de cuatros grupos en lo que respecta a sus períodos de vuelo:

1.- Especies invernales

Pertenecen a este grupo T. cshoenemundi, L. maroccana y L. stupeningi.

2.- Especies y subespecies primaverales

En este grúpo englobamos P. meyeri. P. $p$. asturica, A. s. guadarramensis, N. c. umbrosa. I. bipartita, 1. grammatica sp. 111, P. dispar, Martba nea sp.. P. madritensis y S. torrentium.
De la comparación de nuestros datos con los de otros autores (Aubert, 1963; Terra, 1979 y Berthélemy y Terra, 1980) puede deducirse que:

A.- Existe una tendencia de las especies primaverales del Tambre a aparecer más precozmente que en otras zonas de la Península Ibérica.

B. Las especies otoñales presentan un período de vuelo similar en el Tambre y en el resto de la Península.

Estas conclusiones están de acuerdo con las observaciones de otros autores (Brinck, 1949 y Berthélemy, 1966) que señalan la circunstancia de que las especies primaverales aparecen más pronto en laś zonas bajas que en las altas, mientras que el factor altitud no afecta a las especies otoñales. Hay que señalar que la cuenca del Tambre es una zona más baja 


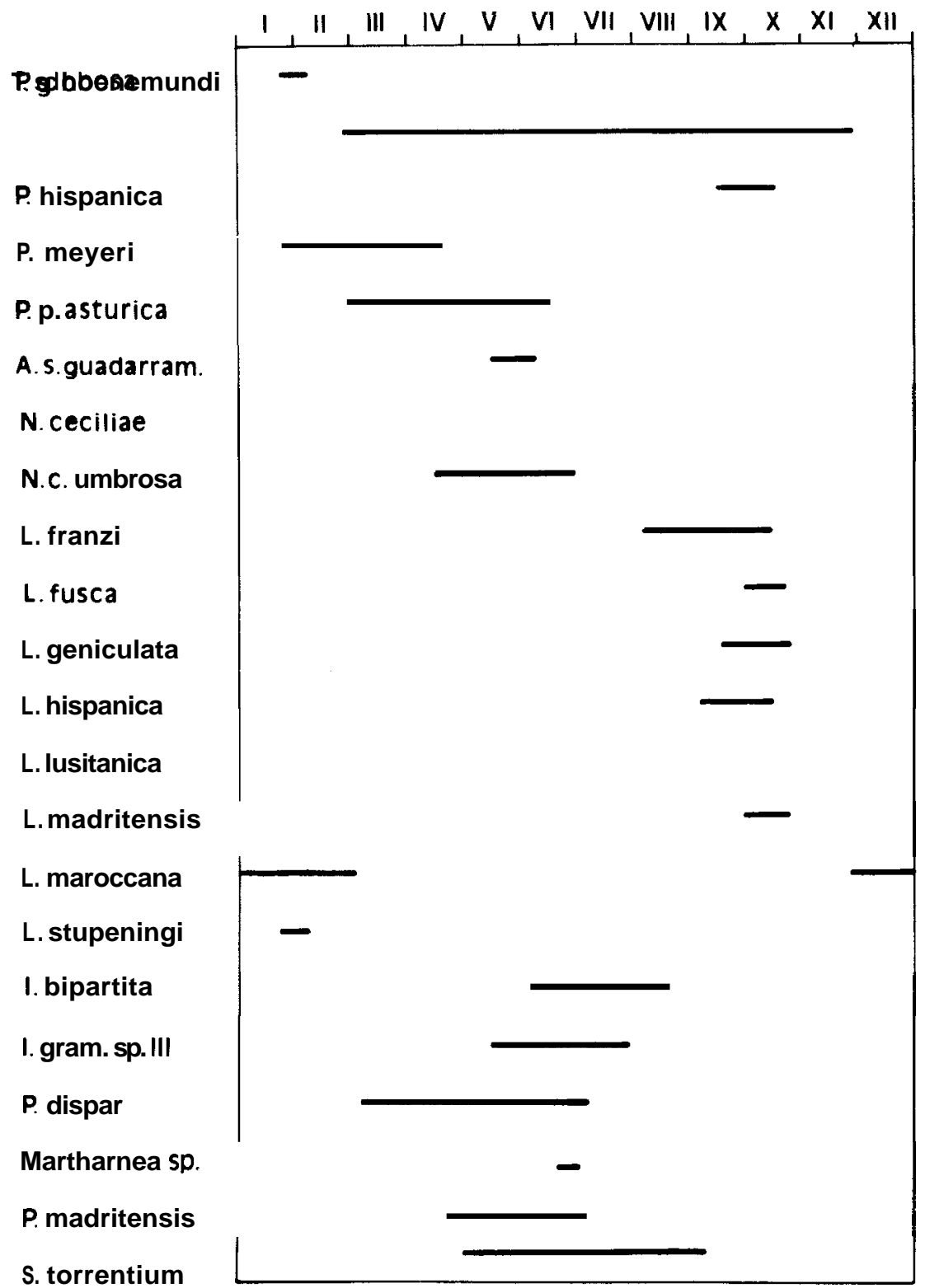

Figura 3.- Períodos de vuelo de los Plecópterten en la cuencal del Tambre Flight periods of the Plecoptera in the Timbre watershed

que la mayoría de las áreas muestreadas hasta la fecha en la Península Ibérica.

\section{BIBLIOGRAFIA}

Aubert, J. 1963. Les Plécoptères de la Peninsule Iberique. Eos, 39 23-107.

Berthélerny, C. 1966. Recherches écologiques et biogéographiques sur les Plécoptères et Coléoptères d'eau courante (Hydraena et Elmintidae) des Pyrénées. Annls Limnol., 2 227-458
Berthélerny, $\boldsymbol{C}$. y Laur, C. 1976. Plécopteres aquatıques du Lor (Massif Central Francais) Annls Limnol., 11(3): 263-285

Berthélemy, C. y Terra, L.S. Whytton da 1980. Plécopteres du Portugal. Annls Limnol 16 (2): 159-182

Brinck, P. 1949. Studies on Swedish Stoneflies (Plecoprera) Opusc Ent., Suppl XI, Lund: $250 \mathrm{p}$

Terra L.S. Whytton da 1979. Note on the portuguese Plecoptera. Gewäss Abwäss, 64: 60-68.

Zwick, P. 1972. Plecoptera (Ins.) aus dem Mittelmeegebiet, vor allem aus Portugal und Spanien. Cienc.Biol., 1: 7-17. 